\title{
RursuSpicae
}

Transmission, réception et réécriture de textes, de I'Antiquité au Moyen Âge

$2 \mid 2019$

Le Physiologus. Manuscrits anciens et tradition médiévale

\section{La tradition manuscrite du Physiologus grec au miroir de témoins conservés en France et en Italie : réflexions pour une étude comparée}

The Manuscript Tradition of the Greek Physiologus According to the Manuscripts Preserved in France and in Italy: some Considerations for a Comparative Study

Adele Di Lorenzo

\section{(2) OpenEdition}

Journals

Édition électronique

URL : http://journals.openedition.org/rursuspicae/666

DOI : $10.4000 /$ rursuspicae.666

ISSN : 2557-8839

Éditeur :

Université Nice-Sophia Antipolis, IRHT - Institut de recherche et d'histoire des textes

Référence électronique

Adele Di Lorenzo, «La tradition manuscrite du Physiologus grec au miroir de témoins conservés en France et en Italie : réflexions pour une étude comparée », RursuSpicae [En ligne], 2 | 2019, mis en ligne le 20 décembre 2019, consulté le 29 mars 2021. URL : http://journals.openedition.org/rursuspicae/ 666 ; DOI : https://doi.org/10.4000/rursuspicae.666

Ce document a été généré automatiquement le 29 mars 2021.

RursuSpicae 


\section{La tradition manuscrite du Physiologus grec au miroir de témoins conservés en France et en Italie : réflexions pour une étude comparée}

The Manuscript Tradition of the Greek Physiologus According to the

Manuscripts Preserved in France and in Italy: some Considerations for a Comparative Study

Adele Di Lorenzo

1 La tradition manuscrite relative au Physiologus grec est toujours d'un grand intérêt; le sujet, vu sous différents angles selon les intérêts littéraires, textuels, paléographiques, codicologiques et d'histoire de l'art, a accumulé à son avantage une riche bibliographie, qui vient de s'enrichir de récentes et fructueuses recherches ${ }^{1}$. Mon intérêt pour la tradition manuscrite grecque du Physiologus est né d'une collaboration dans le cadre d'un projet de catalogage et de numérisation ${ }^{2}$ qui a prévu une première phase de description paléographique et codicologique des manuscrits grecs de la deuxième recension, selon la structure stemmatique formulée par Sbordone ${ }^{3}$. Dans le contexte de cette première expérience de catalogage raisonné, j'ai été confrontée à nombre de cas qui ont suscité mon intérêt et ma curiosité ; les sondages effectués jusqu'à présent m'ont permis de me faire une bonne idée de la constellation que forment les recensions $\mathrm{du}$ Physiologus. En termes chronologiques, cela va des fragments les plus anciens conservés, que l'on peut estimer du XI ${ }^{e}$ siècle, à un manuscrit daté de 1585 .

2 C'est précisément en partant des témoins les plus récents que j'ai mené mes recherches. Le manuscrit Paris, Bibliothèque nationale de France, grec 834, conçu par Gonzalo Ponce de León, représente un point d'arrivée important pour la tradition manuscrite et surtout il constitue la base de l'édition imprimée préparée par le même 
Gonzalo. Francesco Sbordone a placé ce manuscrit à la fin de ses recensions, dans l'appendice $\mathrm{g}$, sous la remarque générale: per infima quoque graecitatis tempora propagationem testantur ${ }^{4}$.

3 Le même codex a représenté le point de départ de mes réflexions sur un groupe de quatre manuscrits produits à Rome et aujourd'hui conservés dans diverses bibliothèques italiennes. Ils avaient déjà attiré l'attention de l'historienne Xenia Muratova, qui dans un article paru dans le Jahrbuch der Österreichischen Byzantinistik de $1982^{5}$, identifiait un groupe de manuscrits de la deuxième recension, tous à peu près contemporains et constituant indubitablement des commandes de luxe de la part de la Curie romaine. Je n'entrerai pas dans les détails du contexte historique et culturel de la production des manuscrits liée au milieu de la cour papale : la place que tiennent, dans la renaissance des lettres grecques en Occident, les savants et les copistes d'origine byzantine est bien connue ; c'est à eux que s'adressaient, au Xvi ${ }^{\mathrm{e}}$ siècle, les papes et les cardinaux-bibliothécaires pour accroître les fonds grecs de la Bibliothèque Vaticane.

Mes recherches sont fondées sur un certain nombre d'observations qui se recoupent : ces manuscrits sont des copies tardives (xvie siècle) relevant de la seconde recension du Physiologus, que Sbordone qualifiait de «byzantine » et dont la rédaction est attribuée par la tradition manuscrite à Épiphane de Chypre. Plus précisément, ils font partie du troisième type, c'est à dire le type $\mathrm{c}$, au sein de la recensio quarta ${ }^{6}$, et, comme l'avaient déjà noté Francesco Sbordone et après lui Xenia Muratova, ils présentent tous les mêmes divisions du texte et la même disposition des illustrations. En mettant en relation les caractéristiques paléographiques et codicologiques de ce petit groupe de manuscrits, on parvient à la conclusion qu'ils sont le produit d'un même milieu culturel et politique qui associe Rome à Venise, mais aussi à Paris, localisations actuelles de témoins intrinsèquement liés dès l'origine.

5 Le groupe italien est formé de quatre manuscrits, dont les trois premiers font partie de la famille $\mathrm{H}$ :

1. Città del Vaticano, Biblioteca Apostolica Vaticana, Barb. gr. 438

2. Città del Vaticano, Biblioteca Apostolica Vaticana, Ott. gr. 354

3. Milano, Biblioteca Ambrosiana, \& 143 sup (Martini-Bassi 775)

4. Venezia, Biblioteca Nazionale Marciana, Marc. gr. IV, 35 (1383) ${ }^{7}$

6 La méthodologie de description paléographique ici adoptée suit le schéma mis au point par Paul Canart dans son travail, demeuré parfaitement valide, sur le copiste Emmanuel Provataris, originaire de Crète, scriptor graecus à la bibliothèque Vaticane dans le troisième quart du XVI ${ }^{e}$ siècle ${ }^{8}$. La lecture des textes tirés des quatre manuscrits suscite d'intéressantes questions sur la position des manuscrits à l'intérieur du stemma codicum établi par Sbordone, et depuis lors inchangé. En premier lieu, il est possible de supposer, sur la base des résultats de Sbordone ${ }^{9}$, que le texte de la recension $\mathrm{H}\left(\mathrm{H}^{\prime}, \mathrm{H}^{\prime \prime}\right.$, $\left.\mathrm{H}^{\prime \prime}\right)$ transmet un type de rédaction mixte qui nécessite des approfondissements. L'examen philologique de cette petite famille de manuscrits romains nécessite une recherche détaillée ; celle-ci sera encore plus intéressante si elle peut s'appuyer sur une relecture attentive de tous les manuscrits de la rédaction byzantine. C'est la raison pour laquelle il faut souhaiter des travaux parallèles et convergents de la part d'autres spécialistes.

7 Mon travail se limite à examiner la question sur la base d'une enquête privilégiant l'aspect paléographique et codicologique. Jusqu'à présent, les résultats que j'ai obtenus 
sont assez clairs : d'une part, ils confirment ce qui a déjà été dit sur l'histoire de l'illustration du Physiologus, et d'autre part, ils mettent au centre de l'attention un milieu de scribes et collaborateurs qui ont prêté leurs talents à la copie de ces manuscrits répondant pour ainsi dire à la vogue romaine de l'époque. En détail, il est possible de définir avec un bon degré d'approximation le contexte de production de ces artefacts. Ces considérations sont fondées sur des examens comparatifs avec plusieurs manuscrits, qui m'ont conduite à ces premières réflexions.

\section{Les manuscrits}

\section{Barb. gr. 438}

Le Barberini grec 438 n'a pas encore été catalogué et l'on ne dispose que d'une description succincte par le bibliothécaire de la famille Sante Pieralisi; une reproduction numérique a été mise en ligne sur le site de la Bibliothèque Vaticane. Le manuscrit est de format in-quarto $(226 \times 168 \mathrm{~mm})$, formé de 45 feuillets d'un papier occidental plutôt médiocre, épais et assez rêche, et que l'encre, en s'oxydant, a détérioré. La reliure, moderne, est datée de 1828 sur le contreplat, de la main de Luigi Maria Rezzi, bibliothécaire des Barberini. Le manuscrit est formé de six cahiers, tous des quaternions sauf le troisième, qui est un ternion : $1 \times 8-1$ (7) $1 \times 8$ (15) $1 \times 6(21) 3 \times 8$ (45).

9 Les cahiers ont une signature en chiffres arabes au verso du dernier feuillet; la signature figure aussi parfois sur le recto du feuillet suivant: ff. $7 \mathrm{v}-8 \mathrm{r} ; 15 \mathrm{v}-16 \mathrm{r}$; $21 \mathrm{v}-22 \mathrm{r} ; 29 \mathrm{v}-30 \mathrm{r} ; 37 \mathrm{v}-38 \mathrm{r}$.

10 Le dernier cahier est signé avec ce qui semble être une croix, au feuillet $38 \mathrm{v}$ dans la marge supérieure. Le f. $45 \mathrm{v}$ présente la formule conclusive suivante, commune à la rédaction $\mathrm{H}^{10}$ : $\Theta \varepsilon ́ \lambda$

11 Les manuscrits copiés par des contemporains romains de Provataris utilisent à plusieurs reprises le même papier. Le papier du Barberini grec 438 présente deux filigranes :

- ff. $23+28 ; 25+26 ; 32+35$ : un homme en prière devant la croix. Il correspond au filigrane reproduit par Canart, Provataris 41, p. 223 (datation : 1542-1572).

-ff. 41+42 : étoile dans un cartouche inscrit dans un cercle (Briquet 6097 a. 1566-1567); il semble correspondre à Canart, Provataris 26 , qui se situe dans la période 3-3b de l'activité du copiste crétois, soit les années 1559-1561.

12 Au verso du feuillet de garde (f. Iv), un dessin à la plume représente saint Épiphane de Chypre. Le texte s'ouvre avec le titre mentionnant comme auteur saint Épiphane:

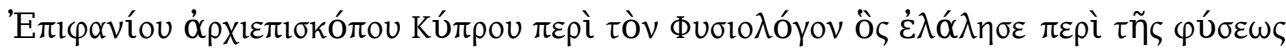

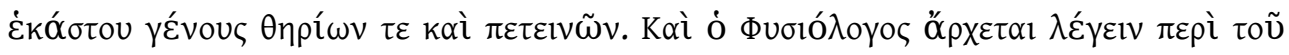

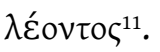

13 L'analyse attentive du manuscrit met en évidence la présence de portions de texte mélangées entre les trois rédactions indiquées par Sbordone, la $1^{\text {ère }}$, la plus ancienne, la $2^{\mathrm{e}}$ ou rédaction byzantine, et la $3^{\mathrm{e}}$ ou rédaction basilienne. Voici la structure du texte et la correspondance avec les rédactions transmises : 


\begin{tabular}{|c|c|c|c|c|c|}
\hline Folia & Contenu & Rédactions & Folia & Contenu & Rédactions \\
\hline ff. $1 r-5 r$ & 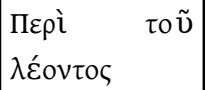 & $1^{\text {ère }} ; 2^{\text {e }} ; 3^{\mathrm{e}}$ & $\begin{array}{l}\text { ff. } \\
23 v-25 v\end{array}$ & 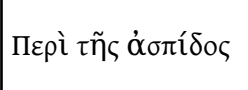 & $2^{\mathrm{e}}$ \\
\hline ff. $5 r-6 v$ & 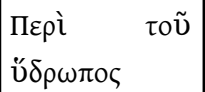 & $2^{\mathrm{e}}$ & $\begin{array}{l}\text { ff. } \\
25 v-26 v\end{array}$ & 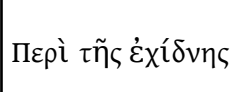 & $2^{\mathrm{e}}$ \\
\hline ff. $6 v-8 v$ & 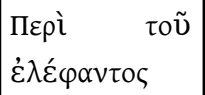 & $\begin{array}{l}2^{\mathrm{e}} \text { mélangée avec la } \\
1^{\text {ère }}\end{array}$ & $\begin{array}{l}\text { ff. } \\
26 v-28 r\end{array}$ & 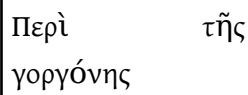 & $\begin{array}{l}2^{\mathrm{e}} \quad \text { mélangée } \\
\text { avec la } 1^{\text {ère }}\end{array}$ \\
\hline $\begin{array}{l}\text { ff. } \\
8 v-10 r\end{array}$ & 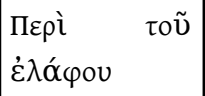 & $2^{\mathrm{e}}$ & $\begin{array}{l}\text { ff. } \\
28 r-30 v\end{array}$ & 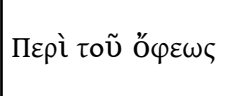 & $\begin{array}{l}1^{\text {ère }} \quad \text { et } \quad 2^{\mathrm{e}} \\
\text { mélangées }\end{array}$ \\
\hline $\begin{array}{l}\text { ff. } \\
10 r-11 v\end{array}$ & 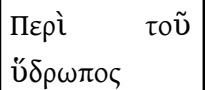 & $2^{\mathrm{e}}$ & $\begin{array}{l}\text { ff. } \\
30 v-32 v\end{array}$ & 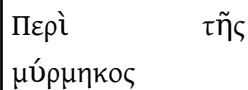 & $1^{\text {ère }}$ \\
\hline $\begin{array}{l}\text { ff. } \\
11 v-12 v\end{array}$ & 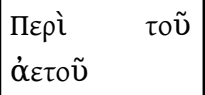 & $2^{\mathrm{e}}$ & $\begin{array}{l}\text { ff. } \\
32 v-33 r\end{array}$ & 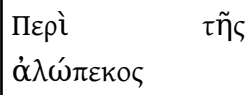 & $2^{\mathrm{e}}$ \\
\hline $\begin{array}{l}\text { ff. } \\
12 v-15 r\end{array}$ & 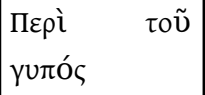 & $2^{\mathrm{e}}$ & $\begin{array}{l}\text { ff. } \\
33 r-34 r\end{array}$ & 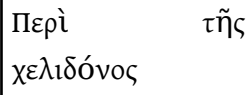 & $2^{\mathrm{e}}$ \\
\hline $\begin{array}{l}\text { ff. } \\
15 r-16 v\end{array}$ & 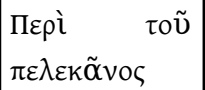 & $2^{\mathrm{e}}$ & ff. $34 \mathrm{rv}$ & 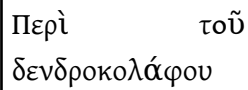 & $1^{\text {ère }}$ \\
\hline $\begin{array}{l}\text { ff. } \\
16 v-18 r\end{array}$ & 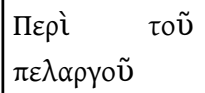 & $2^{\mathrm{e}}$ & $\begin{array}{l}\text { ff. } \\
34 v-35 v\end{array}$ & 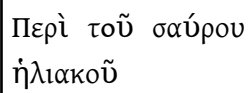 & $1^{\text {ère }}$ \\
\hline ff. $18 \mathrm{rv}$ & 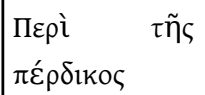 & $2^{\mathrm{e}}$ & $\begin{array}{l}\text { ff. } \\
35 v-36 v\end{array}$ & 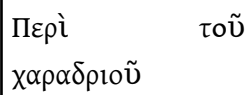 & $1^{\text {ère }}$ \\
\hline $\begin{array}{l}\text { ff. } \\
18 v-19 v\end{array}$ & $\begin{array}{ll}\text { Пврі̀ } & \tau \tilde{\eta} \varsigma \\
\tau \rho v \gamma o ́ v o \zeta & \end{array}$ & $2^{\mathrm{e}}$ & $\begin{array}{l}\text { ff. } \\
36 v-37 r\end{array}$ & 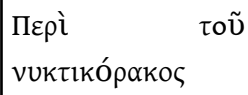 & $2^{\mathrm{e}}$ \\
\hline $\begin{array}{l}\text { ff. } \\
19 v-20 v\end{array}$ & 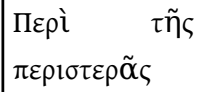 & $2^{\mathrm{e}}$ & $\begin{array}{l}\text { ff. } \\
37 r-39 r\end{array}$ & 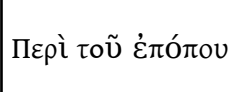 & $2^{\mathrm{e}}$ \\
\hline $\begin{array}{l}\text { ff. } \\
20 v-22 r\end{array}$ & 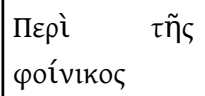 & $\begin{array}{l}2^{\mathrm{e}} \text { avec coupures par } \\
\text { rapport à Sbordone }\end{array}$ & ff. $39 \mathrm{rv}$ & 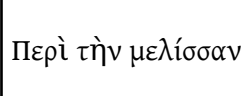 & $2^{\mathrm{e}}$ \\
\hline $\begin{array}{l}\text { ff. } 22 r- \\
23 r\end{array}$ & 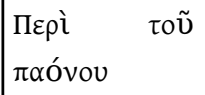 & $2^{\mathrm{e}}$ & $\begin{array}{l}\text { ff. } \\
39 v-40 r\end{array}$ & 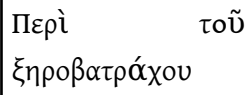 & $2^{\mathrm{e}}$ \\
\hline \multirow[t]{2}{*}{$\begin{array}{l}\text { ff. } \\
23 r-23 v\end{array}$} & 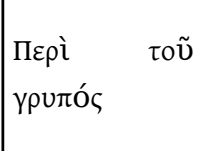 & $2^{\mathrm{e}}$ & $\begin{array}{l}\text { ff. } \\
40 r-43 r v\end{array}$ & 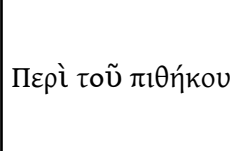 & $\begin{array}{l}\text { Appendix } \\
\text { Sbordone } \\
\text { pp. } 318-319\end{array}$ \\
\hline & & & $\begin{array}{l}\text { ff. } \\
43 v-45 v\end{array}$ & 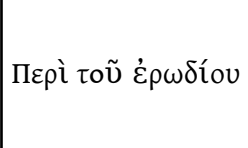 & $\begin{array}{l}\text { Appendix } \\
\text { Sbordone } \\
\text { pp. 319-320 }\end{array}$ \\
\hline
\end{tabular}


L'illustration du manuscrit occupe une position éminente. Selon les réflexions de Xenia Muratova, bien que le manuscrit forme plutôt un ensemble homogène avec les autres du même groupe, la qualité de ses illustrations est plus élevée, trahissant la double influence du style en vogue sous les Paléologue et des usages occidentaux. Une bonne part des types décoratifs de tous les manuscrits est d'origine crétoise, et liée au nom de Théophane de Crète ${ }^{12}$.

L'écriture du manuscrit rappelle celle d'Emmanuel Provataris et de son entourage ; un examen comparatif de l'écriture du Barberini grec 438 et des manuscrits attribuables aux copistes proches de Provataris est donc souhaitable. Dans la présente étude j'ai focalisé mon attention sur des réflexions de nature essentiellement technique.

S'il est relativement facile de distinguer les différents copistes, la manière dont ils collaborent à l'exécution des manuscrits est très peu claire. Certains de ces copistes sont connus : Jean Honorius de Mallia, François Syropoulous, Vivien Brunori. D’autres, dont nous ne connaissons pas le nom, sont néanmoins bien identifiables et leur activité est datable et localisable au milieu du XVI ${ }^{\mathrm{e}}$ siècle à Rome. La question est de distinguer les copies indépendantes réunies sous la même reliure (dès l'origine, parfois) et les manuscrits qui relèvent d'une véritable collaboration, prévue dès le départ: ce n'est évident que lorsque l'on trouve leurs mains dans une seule et même œuvre. Paul Canart a identifié 9 cas avérés de collaboration, et 17 douteux; dans deux de ces derniers, le copiste est connu par ailleurs.

Dans ce contexte, on peut rapprocher d'abord le ms. Barberini (B) de l'entourage de Provataris $(\mathrm{P})$ d'après les critères suivants :

\section{Caractéristiques générales}

18 Encre noire ; rapport équilibré entre largeur et hauteur des lettres; l'espace entre les lignes donne une impression d'harmonie et d'équilibre; constance dans le rapport entre les traits anguleux et les traits arrondis.

\section{Forme des lettres}

La comparaison entre $\mathrm{B}$ et $\mathrm{P}$ a été menée sur une petite sélection de lettres significatives où les habitudes graphiques du copiste sont particulièrement notables.

- $\alpha$ parfois majuscule mais surtout minuscule

- $\beta$ majuscule à deux petites boucles, minuscule normale

- $\gamma$ minuscule, le jambage incliné à gauche, majuscule tantôt petite, tantôt de grande taille

- $\delta$ minuscule étroit et pointu

- $\varepsilon$ majuscule, tracé d'un seul trait et incliné à gauche

- $\zeta$ P utilise deux formes de zêta, que Canart appelle 'zêta gauche' (la boucle supérieure part de la gauche et monte vers la droite) et 'zêta droit' (la boucle descend de la droite vers la gauche) ; B utilise le zêta gauche

- $\eta$ on trouve les deux formes, majuscule et minuscule; la première est plus fréquente, à la différence de $\mathrm{P}$

- $\theta$ souvent ouvert, rarement fermé

- $\kappa$ majuscule de petit corps, exécuté d'un seul trait

- $\lambda$ majuscule mais aussi minuscule quand il est doublé (f. $5 r$ )

- $v$ en forme de $v$ sauf dans les ligatures classiques $\alpha v, \varepsilon v, \eta v$ 
- $\xi$ P, comme dans le cas de zêta, a deux formes $\xi$ gauche et $\xi$ droit ; B a seulement le $\xi$ droit

- $\pi$ les deux formes ; surtout minuscule dans B

- $\rho$ le jambage a une boucle finale vers la gauche

- $\sigma$ on trouve les deux formes $\sigma / \varsigma$

- $\tau$ forme basse fréquente ; forme haut $\varepsilon \tau$ à partie supérieure arrondie ou anguleuse

- $\varphi$ minuscule, il n'a qu'une boucle fermée

Bon nombre de ligatures correspondent à celles qu'utilise Provataris. Voici quelques exemples : $\varepsilon$ de type archaïque, mais dans B la diphtongue est moins développée en longueur ; $\sigma \tau$ avec $\sigma$ ouvert ; $\tau \alpha, \tau o$, avec la boucle inférieure pointue ou arrondie ; $\pi \tau$; $\rho \circ, \rho \alpha$.

Comme l'a montré Paul Canart, l'écriture d'Emmanuel Provataris n'est pas constante tout au long de sa carrière. En ce qui concerne $B$, je retiens les caractéristiques les plus extrêmes pour évaluer le degré de proximité et d'imitation, qui est peut-être le résultat d'une formation professionnelle. Le manuscrit dû à Provataris qui soit le plus proche graphiquement du Barb. gr. 438 est le Vat. gr. 240, dont l'écriture se situe selon Canart dans la période 3b, c'est-à-dire entre 1557 et 1567.

Quelle est la nature des évolutions de l'écriture de Provataris, et dans quelle mesure l'écriture de B peut-elle lui être comparée ? Les éléments-clés de la comparaison entre les deux écritures sont recueillis dans le tableau suivant. La période prise en compte va de 1550 jusqu'en 1560/1567, sur la base des filigranes identifiés :

\begin{tabular}{|l|l|l|}
\hline$\alpha$ majuscule & B & P \\
\hline$\Xi$ & $\begin{array}{l}\text { Présence plutôt rare début /fin } \\
\text { de ligne }\end{array}$ & Rare entre 1557 et 1567 \\
\hline$\zeta$ à droite & Surtout droit & Gauche à partir de 1556 \\
\hline $\begin{array}{l}\beta \text { à deux petites } \\
\text { boucles }\end{array}$ & Usage fréquent et variable & $\begin{array}{l}\text { Rare jusqu'en } 1548, \text { emploi fréquent à àche } \\
\text { partir de 1552 }\end{array}$ \\
\hline $\mathbf{l}$ souscrit & Jamais & Présence fréquente à partir de 1548 \\
\hline Point sur $\mathbf{l}$ et $\boldsymbol{v}$ & Présence variable & Présence variable \\
\hline Sigma final $\sigma$ & Présence moyenne & Présence moyenne vers 1550 \\
\hline
\end{tabular}

De l'expertise menée sur le Barb. gr. 438, nous pouvons tirer ces premières conclusions :

-L'écriture du Barberini grec 438 est proche de celles de l'entourage de Provataris.

- Le manuscrit utilise le même type de papier, avec le même filigrane, que celui qu'utilisent Provataris et ses proches entre 1556 et 1567, c'est-à-dire durant son activité comme scriptor. Concrètement, cela implique que la copie du Physiologus contenue dans le Barb. gr. 438 a été faite à Rome. 
- Le texte du Physiologus présent dans le Barb. gr. 438 est une variante de la deuxième recension, selon la typologie du stemma de Sbordone, mais, plus précisément, il relève d'une recension romaine.

- Les illustrations, selon les spécialistes, témoignent d'une inspiration crétoise alors à la mode dans les cercles romains.

- Certaines sections du texte ont été signalées par des lignes verticales, peut-être dans l'optique d'une nouvelle copie.

\section{Ott. gr. 354}

Le manuscrit Ott. gr. 354 est très bien connu; il est attribué à la main de Manuel Malaxos $^{13}$. Ce dernier, né à Nauplie, capitale du Péloponnèse, au début du XVI ${ }^{\mathrm{e}}$ siècle,

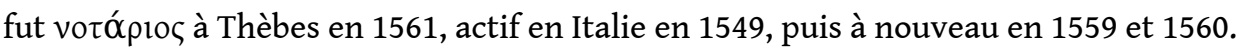

les manuscrits attribués à la main de Malaxos et contenus dans l'actuel fonds des Ottoboni grecs figurent déjà dans l'inventaire de la bibliothèque du cardinal Guglielmo Sirleto, né en 1514 à Guardavalle en Calabre, alors dans le royaume de Naples, et mort le 6 octobre 1585 à Rome. Il fut fait cardinal par Pie IV lors du consistoire du 12 mars 1565, puis nommé évêque de San Marco Argentano (en Calabre) en 1566 ; six ans plus tard, Sirleto est nommé cardinal-bibliothécaire. Sa collection de manuscrits grecs et latins a été bien étudiée. Le ms. Vat. lat. 6163 contient l'inventaire le plus riche et le plus complet de ses manuscrits ; le ms. Ott. gr. 354 est décrit au feuillet $96 \mathrm{v}$, sous le numéro 170 des codices theologici.

6 Le manuscrit, daté $\mathrm{du} \mathrm{XVI}^{\mathrm{e}}$ siècle, faisait partie de la bibliothèque Cervini Sirleto, acquise en 1588 par Ascanio Colonna, puis en 1611 par Giovanni Angelo Altemps. La collection Altemps a été vendue en 1690 à Alexandre VIII (Ottoboni), dont la bibliothèque, enrichie par d'autres apports, a été versée à la Vaticane en 1748 par Benoît XIV. Le feuillet de garde conserve une mention de possession : "ex codicibus Joanni Angeli ducis ab Altemps. Ex graeco manuscripto n. 354".

27 Le contenu et les illustrations du manuscrit sont similaires à ceux du Barb. gr. 438. Les cahiers sont signés au recto du premier feuillet, dans l'angle inférieur droit (ff. 1 ; 9 ; $17 ; 25 ; 33 ; 41 ; 49 ; 57 ; 65 ; 73)$. Datation d'après les filigranes : 1557-1560 :

-ff. $1-8 ; 9+16 ; 11+14 ; 27+30 ; 33-40$ : étoile dans un cercle, attestée en 1557-1567 (cf. De Gregorio, Malaxos, n. 70, et Canart, Provataris, n. 26) ; le même filigrane se retrouve dans d'autres mss de Malaxos : Ott. gr. 100 ; Ott. gr. 349.

- ff. 10+15;12-13;17-24;25-26+31-32;28-29; 41-80 : échelle dans un écu sommé d'une croix, 1546-1552 (cf. Briquet 5929 ; De Gregorio, Malaxos, n. 68 ; Canart, Provataris n. 21).

Giuseppe De Gregorio a qualifié l'écriture du manuscrit de «variante posée de Malaxos ${ }^{14}$, et l'a datée des années $1557-1560$. Il parle d'une copie à main posée, calligraphique, à rapprocher des autres manuscrits copiés par Malaxos. L'examen attentif de certaines lettres, $\delta, \zeta, \imath, \lambda$, o, et de certaines ligatures du ' $\tau$ bas' comme $\alpha \tau$, $\kappa \tau$, $\sigma \tau$, renforce l'identification. La rubrication des titres et des initiales est attribuée au même copiste; De Gregorio estime par contre que les illustrations sont d'une autre main, mais proche de celle du ms. Vat. gr. 1188. Pour l'analyse paléographique, je renvoie à De Gregorio, et m'en tiens aux caractéristiques les plus évidentes :

- Présence de $\gamma$ minuscule

- $\theta$ surtout ouvert

- 1 pointé 
$\cdot \mu$ sans ligature

- $v$ toujours lié

- deux versions du $\xi$, gauche et droit

- $\sigma$ final majuscule ou minuscule

\section{Ambr. \& 143 sup.}

Le manuscrit Ambr. \& 143 sup. appartient à la même rédaction romaine; il est d'un intérêt majeur. D'après le catalogue de Martini-Bassi, le manuscrit compte 16 feuillets seulement, précédés d'un et suivis de trois feuillets de garde. Le feuillet de garde supérieur date de la reliure à l'Ambrosienne et donne, de la main d'Antonio Olgiati, professeur de rhétorique auprès du cardinal Borromée, le titre De quorundam animantium natura. Olgiati fut nommé en 1609 premier préfet de la bibliothèque Ambrosienne ; il participa pendant plusieurs années à la rédaction d'inventaires et de catalogues.

Le manuscrit faisait partie de la collection de Gian Vincenzo Pinelli, homme de lettres et bibliophile célèbre, né à Naples en 1535 et mort à Padoue en 1601. Sa bibliothèque, qui était immense, contenait une importante collection de manuscrits, notamment grecs, qui fut à sa mort versée à l'Ambrosienne.

1 On trouve également, f. 1r, une table partielle du manuscrit. Ce dernier figure dans l'inventaire manuscrit de Giovanni Donato Ferrari (Ambr. Z 13 inf., f. 13v).

Le manuscrit est constitué de deux quaternions, avec traces d'une foliotation originaire sur laquelle est visible la foliotation moderne ${ }^{15}$. En voici le contenu:

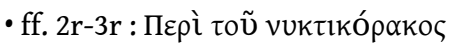

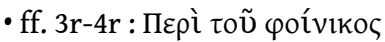

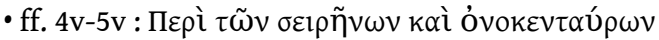

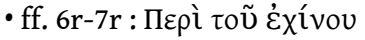

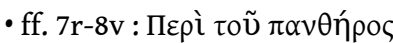

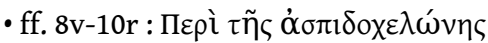

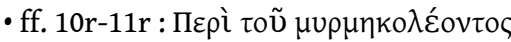

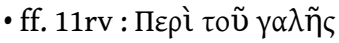

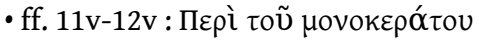

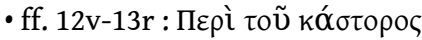

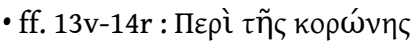

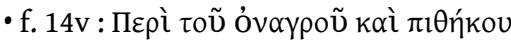

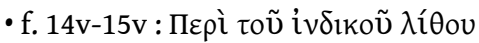

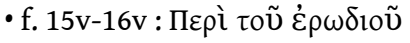

\section{Filigranes}

Ff. $2+9 ; 4+7 ; 11+12 ; 15+16$ : fleur de lis dans un cercle sommé d'une étoile (cf. Briquet 7318-9 - Pise 1581-84 et 1592 ; Canart, Provataris n. 35).

Le manuscrit de l'Ambrosienne présente une écriture similaire à celle de Manuel Malaxos, en particulier elle peut être rapprochée de celle du Vat. gr. 689; dans ces deux cas, l'écriture est très caractéristique: influence de la Fettaugenmode, hampes exubérantes, ductus irrégulier au sein d'un même mot. 


\section{Caractéristiques générales}

- Copie à l'encre noire, légèrement inclinée sur la droite, de nature calligraphique et assez aérée, avec des variations de module importantes pour certaines lettres, dont $\theta$ et $\sigma$ minuscules, moindres pour les boucles du $\varphi$ et du $\omega$.

- Lettres prolongées sous la ligne d'écriture $: \chi, \eta, \gamma, \varphi$.

- Les lettres majuscules sont fréquentes $; \kappa ; \pi ; \eta \varepsilon$, sont petits et souvent inclinés vers la gauche; grand $\sigma$ lunaire; $v$ en fin de mot, $\gamma$ haut sur la ligne. Le copiste utilise peu d'abréviations, largement réservées aux nomina sacra et à la diphtongue ov.

\section{Forme des lettres}

- $\alpha$ petit et arrondi

- $\beta$ à boucles ouvertes, ou bien grandes et fermées

- $\gamma$ minuscule, à jambage pointu (avec ou sans boucle)

- $\delta$ minuscule, boucle inclinée vers la gauche

- $\varepsilon$ majuscule, de deux types: l'un petit et incliné à gauche, l'autre grand, utilisé pour les ligatures

- $\zeta$ à gauche, le trait final se prolonge sur la ligne

- $\theta$ minuscule ouvert et large

- $k$ surtout majuscule

$\cdot \lambda$ minuscule

- $v$ minuscule en $v$, parfois majuscule

- $\xi$ droit

- $\pi$ majuscule et petit

- $\sigma$ de deux types, l'un en $v$, l'autre en s large

- $\tau$ avec la barre à gauche

- $\varphi$ avec le trait vertical se recourbe vers la gauche

- $\chi$ de forme baroque avec les jambages inférieurs très développés.

Certaines ligatures du manuscrit de Milan révèlent une forte tendance à l'exubérance. L'écriture est très organisée, mais renonce en même temps à la sobriété et emploie des formes baroques et de chancellerie, très élégantes. Voici des exemples de ligatures parmi les plus intéressantes, qui rappellent celles qu'a répertoriées De Gregorio chez Manuel Malaxos :

-1. $\alpha \xi$ De Gregorio, 48

-2. $\varepsilon v / \varepsilon \alpha v$ De Gregorio, 50

- 3. $\sigma \tau \varepsilon \rho$ De Gregorio, 55

- 4. $\theta \varepsilon \rho$ De Gregorio, 51

-5. $\delta_{1} \alpha$ De Gregorio, 49

-6. $\pi \alpha / \pi \alpha \rho$ De Gregorio, 53

Il y a tout lieu de penser que le ms. de Milan a lui aussi été copié à Rome ; le papier et ses filigranes sont identiques, avec une datation entre 1560 et 1580 , à ceux que l'on trouve dans les manuscrits de Malaxos et l'écriture même se révèle très proche de celle de ce dernier. Les illustrations manquent, mais leur emplacement a été préparé.

Les sections du Physiologus contenues dans le manuscrit de Milan appartiennent au $\mathrm{III}^{\mathrm{e}}$ type et à la $\mathrm{IV}^{\mathrm{e}}$ recension et font partie du genre a selon Sbordone ${ }^{16}$. Ces divisions sont 


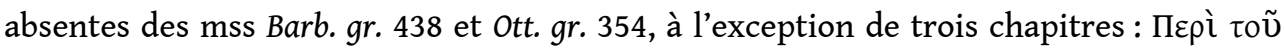

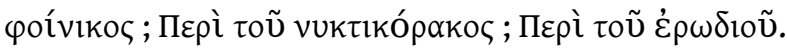

Depuis les travaux de De Gregorio, l'activité de Malaxos est localisée, comme celle de Provataris, au sein d'un cercle privilégié de copistes gravitant autour de la curie romaine ${ }^{17}$. L'écriture grecque qu'il utilise tend (et le manuscrit de Milan le confirme) à varier le module des lettres, phénomène souvent associé à une audace plus grande dans le ductus, qui se révèle plus rapide. Comme l'explique De Gregorio, ce trait est lié à l'expérience professionnelle du copiste, qui a longtemps exercé comme notaire. Au vu des premières constatations, l'Ambrosiano est à rapprocher d'un point de vue paléographique du Vat. gr. 689.

\section{Marc. gr. IV 35}

Le ms. grec IV, 35 de la Bibliothèque Marciana de Venise est tenu pour l'archétype de la recension $\mathrm{H}$ de Sbordone. C'est un manuscrit du XVI ${ }^{\mathrm{e}}$ siècle, sur papier occidental assez foncé, de dimensions $210 \times 161 \mathrm{~mm}$ (justification : 141 x $40 \mathrm{~mm}$ par colonne). La copie est effectuée à raison de 13 ou 14 lignes par page. Le manuscrit compte 87 feuillets précédés d'un feuillet de garde; les cahiers sont tous quaternions. La foliotation est postérieure. Au f. 80, le dernier chapitre a été signé, en grec, $1 \alpha$ ’. Les illustrations sont numérotées dans la marge supérieure (49).

Le manuscrit provient de la famille Nani de San Trovaso, qui le possédait au milieu du $\mathrm{XVII}^{\mathrm{e}}$ siècle. La collection familiale, enrichie surtout par les frères Bernardo (1712-1761) et Giacomo (1725-1797), passa au XIX siècle à la Bibliothèque Marciana.

\section{Filigranes}

-ff. $27+28 ; 35+36 ; 75+76$ : tête de bœuf (Briquet 1447o a. 1539 De Gregorio, Malaxos, n. $71 / 72)$

- ff. 25-30; 58-61; 81-86 : ancre inscrite dans un cercle sommé d'une étoile (Briquet n. 480 a. 1508, De Gregorio, Malaxos, 2a)

41 En plus des illustrations, le manuscrit est rubriqué, pour les titres et les initiales, avec une encre de couleur ocre, bien attestée chez Malaxos et dans son entourage. Les illustrations, pour ce qu'elles représentent et pour leur disposition, sont similaires à celles du Barb. gr. 438 et de l'ott. gr. 354.

\section{Forme des lettres}

Le manuscrit a été copié avec une encre très noire; l'écriture présente des caractéristiques communes avec la main d'un collaborateur de Malaxos, que De Gregorio appelle le "collaborateur phi ", à cause de la boucle triangulaire du phi inclinée vers la gauche. Pour l'identification de la main, je me suis fondée sur la comparaison du ms. de Venise avec le f. 22v du Vat. gr. 834 et les ff. 10r-11r de l'Ott. gr. 260. La liste suivante résume les caractéristiques communes les plus intéressantes :

- $\alpha$ minuscule, petit

- $\beta$ minuscule à boucle inférieure surdimensionné

- $\gamma$ à jambage pointu

- $\varepsilon$ majuscule, deux types : un petit, l'autre grand en ligature

- $\varepsilon v$ avec epsilon triangulaire 
- $\varepsilon \pi$ incliné

- $\zeta$ traverse inférieure allongé

- $\eta$ majuscule, petit

- $\theta$ ouvert et large ou fermé et étroit

- $\kappa \alpha 1$ tachygraphique et de grande dimension

- $\mu$ majuscule et minuscule

- $v$ en finale majuscule, arrondi et surdimensionné

- o large

- $\pi$ majuscule, légèrement incliné à droite

- $\rho$ incliné à gauche

- $\sigma$ élargi et arrondi

$\cdot \tau+$ o o suscrit

- $v$ petit si isolé et grand en ligature

- $\varphi$ boucle pointue et surdimensionnée

- $\chi$ gauche à jambages inférieurs prolongés sous la ligne

En général, l'écriture rappelle l'écriture posée de $\operatorname{Malaxos}^{18}$, mais elle est plus libre et plus baroque. Elle se rattache plus à la stylisation baroque du XVI siècle qu'à la tendance, d'égal succès, du style des Hodèges. L'une des caractéristiques majeures de ce phénomène est la "Fettaugenmode ", c'est-à-dire le fait de surdimensionner certaines lettres de forme ronde $(0, \sigma, \omega, \theta)$; il s'en dégage l'impression que l'élément majeur est le contraste, comme dans bon nombre des expériences graphiques grecques du XVI siècle. La diversité d'activité des copistes, qui ont souvent servi dans plusieurs chancelleries, tant en Occident, qu'en Orient, est à la base de ce courant graphique.

\section{Traits communs et divergences}

Les recherches sur l'activité des copistes (Provataris et Malaxos pour ce qui nous concerne), des possesseurs et des marchands de manuscrits concernent particulièrement les réseaux de copistes, d'élèves, de collaborateurs qui les entourent. Canart et De Gregorio ont bien montré qu'il n'a pas existé de scriptorium stricto sensu autour de Provataris ou de Malaxos, mais des cercles, des relations de collaboration, où, dans tous les cas, ces deux personnages jouaient un rôle central, assez proche de celui d'un chef d'atelier. L'une des preuves de cette organisation est que les manuscrits réalisés dans leur entourage portaient la «marque » de Provataris ou de Malaxos; ce dernier, par exemple, copiait lui-même les titres, dans une encre très reconnaissable, de couleur ocre, caractéristique, précisément, de sa production.

Pour terminer ce bref examen des expériences graphiques que présentent ces trois manuscrits, comparons leur contenu, pour faire ressortir ce qui les rapproche et ce qui les distingue.

Le tableau qui suit présente les sections du Physiologus que l'on trouve dans ces quatre témoins. En gras sont indiquées les sections communes :

On voit bien que le manuscrit de Milan, peut-être copié par Malaxos, plus ou moins contemporain des trois autres, est organisé complètement différemment : il pourrait s'agir au départ d'un livret de deux fascicules contenant une addition étrangère aux trois autres manuscrits, servant un autre projet, ou constituant la partie d'un manuscrit jamais achevé. Aucun des trois autres manuscrits romains ne porte la trace 
d'une lacune matérielle où il faudrait insérer le texte du ms. de l'Ambrosienne. L'Ott. gr. 354 et l'Ambr. \& 143 sup. peuvent certes être tous les deux de la main de Malaxos, mais la paléographie montre qu'ils ont été copiés à des moments différents, probablement à une dizaine d'années d'écart. Ces manuscrits ont également des filigranes différents, de datation relativement distincte - mais ce n'est pas un critère décisif, parce qu'un filigrane donné peut être utilisé pendant une période assez longue.

L'analyse du contenu des manuscrits nous apprend que certaines sections, en rouge dans le tableau, sont communes aux trois manuscrits. La question à résoudre et à vérifier est de savoir si Malaxos avait entrepris de copier un autre Physiologus, qu'il n'a jamais achevé.

\section{Remise en contexte et conclusion}

Les éléments techniques développés jusqu'à présent débouchent sur des hypothèses plus générales quant à la façon dont les copistes répondaient à une demande relativement soutenue en manuscrits, sur fond de concurrence croissante de l'imprimé.

Les analyses montrent d'abord que la production de copies du Physiologus se concentrait sur un segment de production, sur un type matériel précis de manuscrits, dont le format et les autres caractéristiques codicologiques étaient bien adaptés à un texte illustré. Sur les méthodes de travail, ensuite, les aperçus ne sont pas moins intéressants : Provataris et Malaxos recouraient à des collaborations pour achever ou réaliser des commandes, qui témoignent du magistère graphique du copiste en chef et de la reconnaissance d'un style propre, adapté aux besoins spécifiques du Physiologus. Pour l'heure toutefois, les paléographes hésitent à parler d'ateliers rigides et identifiables, préférant l'image de cercles de collaborateurs ouverts, qui n'excluent d'ailleurs pas l'existence d'un véritable apprentissage auprès des maitres.

1 Pour en terminer avec la rédaction romaine du Physiologus, il faut prendre en compte deux autres manuscrits, dont l'importance a déjà été soulignée par Muratova : Rome, Biblioteca Vallicelliana, gr. 68 et Biblioteca Casanatense 1700.

Mais il me faut d'abord évoquer le dernier manuscrit du groupe "romain", qui se trouve être le manuscrit préparatoire à l'édition imprimée préparée par l'Espagnol Gonzalo Ponce de León, camérier secret de Sixte Quint (aujourd'hui Bibliothèque nationale de France, grec 834) :

- Papier. ff. A-II et 97 pages. Copié en $1585 .{ }^{19}$ Format moyen. Reliure de maroquin rouge aux armes de Sixte Quint, (Consalus Ponce de), Versiones S. Epiphanii physiologi et S. Petri liturgice.

Cette édition manuscrite contient une introduction et douze chapitres du Physiologus, tous éléments que l'on retrouve dans l'édition imprimée réalisée par Ponce de León à Rome en 1587, sur les presses de Luigi Zanetto et Giacomo Ruffinello. Dans l'introduction, l'éditeur expose les raisons qui l'ont poussé à réaliser son ouvrage, et surtout, il cite les manuscrits de base de son édition, un fait déjà signalé par Sbordone. Ponce de León parle en effet de trois manuscrits, dont l'un, mentionné comme étant à Palerme, n'a pas laissé de trace ; un autre provenait de la bibliothèque du cardinal Sirleto : c'est l'Ottoboni grec 354 , comme l'a déjà vu Muratova ; un troisième enfin provenait de la bibliothèque de Francisco Torres : c'est le manuscrit de la Casanatense. Ce dernier semble jouer un rôle majeur dans la rédaction romaine, même s'il s'agit d'un 
manuscrit très corrigé, marqué par de nombreuses interventions qu'il faudra vérifier soigneusement. Ce manuscrit aussi a été annoté et corrigé par Malaxos.

Sbordone a été très critique à l'égard de Ponce de León : «L'édition publiée en 1587 par l'Espagnol Gonzalo Ponce de León, camérier de Sixte Quint, est une monstruosité. L'éditeur, qui n'a utilisé aucun manuscrit de la rédaction la plus ancienne, et ne connaissait que trois exemplaires du Physiologus byzantin $\mathrm{H}$, s'en est servi de manière parfaitement arbitraire, allant jusqu'à créer de toutes pièces le dernier chapitre de sa collection, du reste réduite et mutilée $\aleph^{20}$.

Ces commentaires font sourire aujourd'hui : mieux que Sbordone, nous savons que Ponce de León n'avait certainement pas l'intention de produire une édition du Physiologus suivant les critères modernes. Son objectif était de diffuser la substance de l'ouvrage, et de le faire avec les manuscrits qui étaient alors à sa disposition. Son édition manuscrite a sa part de mystère: Ponce de León en fait don au pape en y joignant la liturgie de Saint-Pierre, issue d'un autre manuscrit de Sirleto dont il disposait. Cette association laisse perplexe, mais peut s'expliquer. Ponce de León est en effet connu pour avoir travaillé à des ouvrages anti-protestants. Voici ce qu'il écrit à son bienfaiteur, dévoilant son projet apologétique :

Sanctitati tuae non indecorum iudicavi literarius fasciculus fastidire non debet. Addo, pater beatissime, argumenti genus iniocundum cum pias quasdam allegorias atque inversiones contineat, quae erudiendo pro concionibus populo apprime solent esse utile.

Aux années 1540-1590 la commande de copies manuscrites d'ouvrages grecs de la part du pape et de curialistes n'a pas faibli et suscite des réponses spécifiques en matière d'organisation du travail. L'analyse méticuleuse des mains et des manuscrits a déjà brillamment éclairé le sujet à partir de l'étude fondatrice de Paul Canart sur Emmanuel Provataris puis de celle de Giuseppe De Gregorio sur Manuel Malaxos. À leurs côtés s'activent des individus moins connus ou totalement obscurs. On peut encore tourner le regard vers ces sans-grade, engagés dans des degrés très divers de participation occasionnelle ou plus suivie. Les communautés de format, l'originalité du montage textuel comme celle d'une illustration soignée, les spécificités graphiques, tout en nous permettant de serrer les identifications, montrent aussi que Provataris et Malaxos pouvaient réussir à définir un style et un produit attractifs pour l'entourage curialiste. La poignée de manuscrits que j'ai ici passés en revue donnent, parmi bien d'autres, un aperçu de la fortune du Physiologus grec, parvenu sous Sixte Quint au terme d'un voyage aux multiples étapes, et après avoir été jugé suffisamment important pour que des mécènes puissants et des copistes de renom se soient donné la peine de le copier ou de le faire copier à l'apogée de la Renaissance romaine. 


\section{BIBLIOGRAPHIE}

M. L. AGATI, « Pietro Devaris di Corfù, scriba librarius Vaticanus: l'identificazione di uno 'Pseudo-

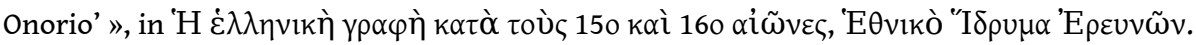

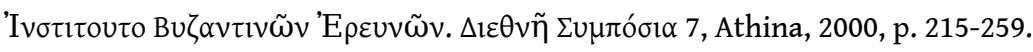

P. CANART, « Les manuscrits copiés par Emmanuel Provataris (1546-1570). Essai d'étude codicologique ", in M. L. AGATI - M. D'AGOSTINo (éd.), Études de paléographie et de codicologie I, Città del Vaticano, 2008, (Studi e Testi, 450), p. 33-166.

G. DE GREGORIO, Il copista greco Manouel Malaxos. Studio biografico e paleografico-codicologico Città del Vaticano, 1991, (Littera Antiqua 8), p. 179-180.

S. LAZARIS, « Un nouveau manuscrit grec illustré du Physiologus : au sujet d'une récente étude sur ce texte », Revue des études byzantines, 58, 2000, p. 279-281.

ID., « Nouvelles perspectives dans l'étude du Physiologus grec et de son illustration », Archimède, 1, 2014, p. 161-163.

ID., Le Physiologus grec, t. 1. La réécriture de l'histoire naturelle antique, Firenze, Sismel, 2016.

X. MURATOVA, « La production des manuscrits du Physiologue grec enluminés en Italie aux XV $\mathrm{X}_{-}$ $\mathrm{XVI}^{\mathrm{e}}$ siècles et leur place dans l'histoire de la tradition de l'illustration du Physiologue », Jahrbuch der Österreichischen Byzantinistik, 32/6, 1982, p. 327-340.

F. SBORDONE, Physiologus, Milano, 1936.

A. ZUCKER, Physiologos. Le bestiaire des bestiaires, Grenoble, 2004.

\section{NOTES}

1. Je cite les travaux les plus récents qui relèvent de plusieurs pistes de recherches : S. LAZARIS, Un nouveau manuscrit grec illustré du Physiologus : au sujet d'une récente étude sur ce texte, REB, 58 (2000), p. 279-281 ; A. ZUCKER, Physiologos. Le bestiaire des bestiaires, Grenoble 2004 ; s. LAZARIS, Nouvelles perspectives dans l'étude du Physiologus grec et de son illustration, Archimède, 1 (2014), p. 161-163 ; ID., Le Physiologus grec, t. 1. La réécriture de l'histoire naturelle antique, Firenze, Sismel, Firenze, 2016. Pour l'édition du texte du Physiologus grec, la plus récente et reconnue reste celle de F. SBORDONE, Physiologus, Milano, 1936. La présente contribution livre les premières conclusions d'une enquête dont Paul Canart a suivi le développement avec son habituelle bienveillance. J'entends la poursuivre et l'enrichir comme il l'aurait souhaité.

2. Le projet 'Physiologus grec on-line', sous la direction de Stavros Lazaris a été conçu au sein de l'Université Paris-Sorbonne et du Labex Resmed (http://www.labex-resmed.fr/le-physiologusgrec-on-line, consulté le $1^{\text {er }}$ décembre 2019).

3. Voir la n. 6.

4. SBORDONE, Physiologus, [voir n. 1], p. XXVIII-XXIX.

5. X. MURATOVA, La production des manuscrits du Physiologue grec enluminés en Italie aux $\mathrm{XV}^{\mathrm{e}}-\mathrm{XVI}^{\mathrm{e}}$ siècles et leur place dans l'histoire de la tradition de l'illustration du Physiologue, JÖB, 32/6 (1982), p. 327-340.

6. F. SBORDONE, Physiologus [voir n. 1], p. LXXXVII. 
7. Selon Sbordone, le manuscrit de la Marciana est l'archétype du groupe, et tous les autres dépendent de lui. Ibid., p. XxIV; CIV.

8. P. CANART, Les manuscrits copiés par Emmanuel Provataris (1546-1570). Essai d'étude codicologique, dans M. L. AGATI - M. D’AGostino [éd.], Études de paléographie et de codicologie (Studi e Testi, 450), I, Città del Vaticano, 2008, p. 33-166.

9. Sbordone affirme que les manuscrits de $\mathrm{H}$ qui sont compris dans la classe $\mathrm{IV}^{\mathrm{me}}$ de la $\mathrm{I}^{\text {ère }}$ rédaction ont toutefois la caractéristique d'être '... typum tamen per se stantem praebet', $\mathrm{F}$. SBORDONE, Physiologus [voir n. 1], p. XLII. Les manuscrits H sont aussi insérés dans la $\mathrm{II}^{\mathrm{e}}$ rédaction, ibid., p. LXXXVII-LXXXVIII.

10. F. SBORDONE, Physiologus [voir n. 1], p. LXXXVIII.

11. Tous les passages tirés des manuscrits ont été normalisés dans la forme du grec classique.

12. X. MURATOVA, La production des manuscrits, [voir n. 2], p. 333.

13. G. DE GREGORIO, Il copista greco Manouel Malaxos. Studio biografico e paleografico-codicologico (Littera Antiqua 8), Città del Vaticano, 1991, p. 179-180.

14. Ibid. p. 116-117.

15. Mon étude plus détaillée sur ce manuscrit est déjà bien avancée et je souhaite la terminer bientôt.

16. F. SBORDONE, Physiologus [voir n. 1], p. LXXXIVII -LXXXVIII.

17. L'activitè de Manuel Malaxos en Italie est située entre 1548 et 1561. Pour tous les détails, voir DE GREGORIO, Il copista greco, p. 19-39.

18. DE GREGORIO, Il copista greco, [voir n. 10] p. 116.

19. La copie de ce manuscrit a été attribuée par M. L. Agati à Pietro Devaris, scriptor à la Vaticane (M. L. AGATI, « Pietro Devaris di Corfù, scriba librarius Vaticanus: l'identificazione di uno 'Pseudo-

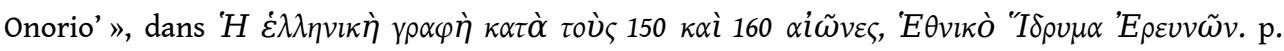
215-259, p. 223 sq.

20. SBORDONE, Ricerche sulle fonti e sulla composizione del Physiologus greco, Napoli, 1936, p. 32.

\section{RÉSUMÉS}

Une nouvelle analyse codicologique et paléographique de quatre manuscrits italiens du Physiologus grec (deuxième recension, troisième type, de la nomenclature de sbordone), composés dans les années 1550-1560 permet de préciser les modalités de leur fabrication. Ces copies de luxe commandées par la Curie romaine ont été en effet réalisées en collaboration par divers membres, plus ou moins identifiés, de l'entourage du scriptor Emmanuel Provataris et de Manuel Malaxos. Bien connus pour leur illustration, ces exemplaires témoignent aussi de la faveur persistante pour le manuscrit, alors même que se prépare, sur leur base, réduite, la première impression de l'ouvrage (1587).

A new codicological and paleographic analysis of four Italian manuscripts of the Greek Physiologus (third type of the second version in Sbordone's nomenclature), compiled in the 1550s-1560s, enables to determine the conditions of their production. These luxurious copies commissioned by the Roman Curia were done in collaboration by various, more or less identified members of the entourage of the scriptor Emmanuel Provataris and of Manuel Malaxos. These 
copies are well known for their illustrations and demonstrate the persistence of preference for the manuscript book, even though the first printing of the work is based on them (1587).

INDEX

Mots-clés : Physiologus grec, manuscrits du Vatican, Emmanuel Provataris, Manuel Malaxos, Ponce de León

Keywords : Greek Physiologus, Vatican Manuscripts, Emmanuel Provataris, Manuel Malaxos, Ponce de León

\section{AUTEUR}

ADELE DI LORENZO

Adele Di Lorenzo est docteur de recherche à l'Université de Sienne, diplômée de l'Ecole Pratique des Hautes Études, et actuellement ingénieur de recherche contractuel à l'Institut de Recherche et d'Histoire des Textes (CNRS). Ses principales publications ont porté sur la paléographie et la diplomatique grecques et latines. 